\title{
Medicines utilization and trends in Sudan between 2006 and 2010
}

\author{
Mohamed A Mousnad ${ }^{1}$, Mohamed Izham M Ibrahim ${ }^{2 \star}$, Subish Palaian ${ }^{3}$ and \\ Asrul A Shafie ${ }^{4}$ \\ ${ }^{1}$ Department of Pharmacy Practice, Faculty of Pharmacy, International University of Africa, Khartoum, Sudan, ${ }^{2}$ Social and \\ Administrative Pharmacy, Clinical Pharmacy and Practice Section, College of Pharmacy, Qatar University, Doha, Qatar, \\ ${ }^{3}$ Department of Pharmacy Practice, Gulf Medical University, Ajman, United Arab Emirates, ${ }^{4}$ Social \& Administrative Pharmacy \\ Discipline, School of Pharmaceutical Sciences, Universiti Sains Malaysia, 11800 Penang, Malaysia
}

${ }^{*}$ For correspondence: Email: mohamedizham@qu.edu.qa

\begin{abstract}
Purpose: This article aims to assess the Sudan National Health Insurance Fund (NHIF) medicines utilization trends and pattern between 2006 and 2010.

Methods: A quantitative descriptive study was conducted to analyse the aggregate spending and utilization data. The aggregate medicine data used in the times series were collected from a large NHIFSudan bulk purchasing system from 2006 to 2010. The Anatomical Therapeutic Chemical (ATC)Classification System and Defined Daily Dose (DDD) methodologies were applied in the study for the period 2006 - 2010, 1st to 4th quarter of each year, ATC classes (14 in all),WHO ABC and VEN (vital, essential, non-essential) categories. Both descriptive and inferential statistics were used to analyse the data.

Results: The total medicine utilization from 2006 to 2010 was 402.62 million DDD with an overall increase of $176.43 \%$ over the period. The medicine classes that accounted for the highest utilization were medicines related to blood and blood-forming organs, followed by the cardiovascular system, general anti-infectives for systemic use, and those pertaining to the alimentary tract and metabolism. Among the most highly used medicines were folic acid, mefenamic acid and amlodipine tablets. Further analysis indicate that there was a statistically significant difference between groups $\{F(4,5270)=$ 54.412, $p<0.001\}$ and weak positive significant correlation between medicine utilization and both population coverage and number of patients $(p<0.001)$.

Conclusion: The results show that medicine utilization in NHIF-Sudan significantly increased from 2006 to 2010. Medicines for blood and blood-forming organs were the highest utilized class of medicines, while folic acid tablet was the most highly used medicine.
\end{abstract}

Keywords: Anatomical, Therapeutic Chemical classification system, Defined daily dose, Medicine utilization, Health insurance, Sudan

Tropical Journal of Pharmaceutical Research is indexed by Science Citation Index (SciSearch), Scopus, International Pharmaceutical Abstract, Chemical Abstracts, Embase, Index Copernicus, EBSCO, African Index Medicus, JournalSeek, Journal Citation Reports/Science Edition, Directory of Open Access Journals (DOAJ), African Journal Online, Bioline International, Open-J-Gate and Pharmacy Abstracts

\section{INTRODUCTION}

According to WHO, information and regular monitoring regarding appropriateness and efficiency of medicines use in developing countries are often lacking [1]. There has been a growing trend of medicine utilization in the NHIFSudan (National Health Insurance Fund-Sudan). In order to gain a better understanding of medicine services' expenditure and trends, the policy makers in the NHIF-Sudan need to track 
the access, utilization and use of medicines for evidence-based decisions.

This research is the first of its kind to present medicine procurement in Sudan using a standard method ATC/DDD system, which is recommended by the WHO. This system allows national and international comparisons of medicine utilization studies [2].The findings are expected to enable the creation of accurate reports and studies to develop more successful medicine services, motivate pharmacy staff in the NHIF to improve and follow the established recommended and international standards, plan for better medicines consumption, improve the quality of statistics on medicines, facilitate national and international comparisons of medicine utilization and suggest a format for application to the entire medicine sector in Sudan.

This article aims to assess the Sudan National Health Insurance Fund (NHIF) medicines utilization trends and pattern during the period 2006 to 2010.

\section{METHODS}

\section{Study design}

This was a mixed observational retrospective and prospective study. A quantitative study was conducted to analyse the aggregate spending and utilization data. Aggregate medicine data managed by a large NHIF-Sudan bulk purchasing system from 2006 to 2010, were collected and used in this study.

\section{Ethical consideration}

The research was approved (ref: 140211206) by the Directorate of Planning \& Research of the NHIF-Sudan headquarters, NHIF-Sudan Committee of Research, the General Director of the NHIF-Sudan and the Minister of Welfare \& Social Security.

\section{Study instrument and tools}

\section{(a) ATC/DDD methodology}

Medicines utilization were expressed as DDDs and number of defined daily doses per 1000 inhabitants per day (DDD/1000/day) or DDD/1000/ Inhabitant /Day(DID) as recommended by the WHO Working Group on Medicine Utilization. DDDs were calculated as per the guidelines for ATC classification and DDD assignment as provided by the WHO Collaborating Centre for Drug Statistics
Methodology [3]. DDDs were calculated as in Eq 1.

Defined Daily Doses $=(Q S) / D$

where $Q$ is quantity of dosage units, $S$ is strength of each dosage unit and $D$ is DDD value assigned by $\mathrm{WHO}$.

DDD/1000/day (DID) for these medicines was calculated as in Eq 2.

Number of DDDs per 1000 inhabitants per day = (QS /T D Ds) 1000

where $Q$ is quantity of dosage units, $S$ is strength of each dosage unit, $T$ is Total sample size (i.e. population covered), $D$ is DDD value assigned by $\mathrm{WHO}$ and $\mathrm{Ds}$ is duration of the study (i.e., usually 1 year, but can be shorter).

The DDDs were compared in order to determine consumption patterns in 2006 and in 2010 and to assess overall consumption trends [4-7].

\section{(b) Therapeutic category analysis}

\section{Operational definition of variables}

The following are variables used in the study:

(i) Dependent variables: Number of DDDs for individual medicines, DDD/1000 day for individual medicines;

(ii) Independent variables: Years (2006-10); $1^{\text {st }}$ to $4^{\text {th }}$ quarter of each year (1-4); ATC classes (14 classes): alimentary tract and metabolism, blood and blood-forming organs, cardiovascular system, dermatologicals, genito-urinary system and sex hormones, systemic hormonal preparations (excluding sex hormones and insulins), anti-infectives for systemic use, antineoplastic and immune-modulating agents, musculo-skeletal system, nervous system, antiparasitic products, insecticides and repellents, respiratory system, sensory organs; various $A B C$ classes [4], and VEN categories [4].

\section{Data management and statistical analysis}

Two independent pharmacists from NHIF-Sudan checked the aggregate spending data obtained from the NHIF database to ensure completeness and reliability of the data. Data were analysed with the computer program Excel and SPSS program (SPSS Inc, released 2009; PASW Statistics for Windows, version 18.0. Chicago: SPSS Inc.). 
Descriptive statistics were used to present the dependent and independent variables. Normality of data was confirmed with a frequency chart (histogram), skewness and kurtosis. The mean and standard deviation were calculated for all normally distributed continuous variables. To describe nominal and ordinal variables, percentages, frequencies and contingency tables were used. The quarterly growth rate of NHIFSudan medicine utilization (2006 to 2010) was presented in a line graph. Pearson correlation, ANOVA, i.e., one-way and two-way were used in the inferential statistical analyses. Tukey's test was applied when the results of ANOVA were statistically significant. The level of statistical significance was set at $p<0.05$ for all analyses.

\section{RESULTS}

\section{Medicine utilization trend in NHIF from 2006 to 2010}

Figure 1 displays medicine utilization trends in NHIF-Sudan between 2006 and 2010. Total utilization via DDD methodology was 402.62 million DDDs. The overall medicine utilization increased by $176.43 \%$ (from 11.46 million DDDs in Quarter 1, 2006 to 31.68 million DDDs in Quarter 1, 2010). The minimum medicine utilization per quarter was 9.29 million DDDs and the maximum recorded was 31.68 million DDDs. The mean utilization was 20.13 million DDDs $( \pm 6.39)$.

\section{Breakdown of medicine utilization by therapeutic class}

Analysis in this section was executed at the three levels of the ATC system, at first level (anatomical main group), at second level (therapeutic use), and at fifth level (subgroup for chemical substances).

\section{(a) Medicine utilization by main therapeutic class}

Table 1 shows the annual medicine utilization by main therapeutic class in NHIF-Sudan for the years from 2006 to 2010. The medicine classes that accounted for the highest utilization were medicines related to blood and blood-forming organs $(27.84 \%)$, followed by the cardiovascular system (20.78\%), general anti-infectives for systemic use $(14.25 \%)$ and the alimentary tract and metabolism (10.97\%). These classes were responsible for most of the medicine utilization (73.84\%).

Medicine utilization change and the percentage contribution of ATC main groups to total utilization from 2006-2010 are presented in Table 3. Among the top four groups, medicines related to blood and blood-forming organs made the largest contribution to the total increase in medicine utilization (130.66\%), followed by the musculo-skeletal system (24.57\%), general antiinfectives for systemic use $(11.42 \%)$ and the respiratory system $(4.16 \%)$. These four classes were responsible for most of the total increase in medicine utilization (170.81\%) (Table 3).

In terms of average annual growth rate for the study period 2006- 2010, medicines for the musculo-skeletal system had the highest average $(250.93 \%)$, and those for the respiratory system had the lowest average $(-27.37 \%)$ (Table 2).

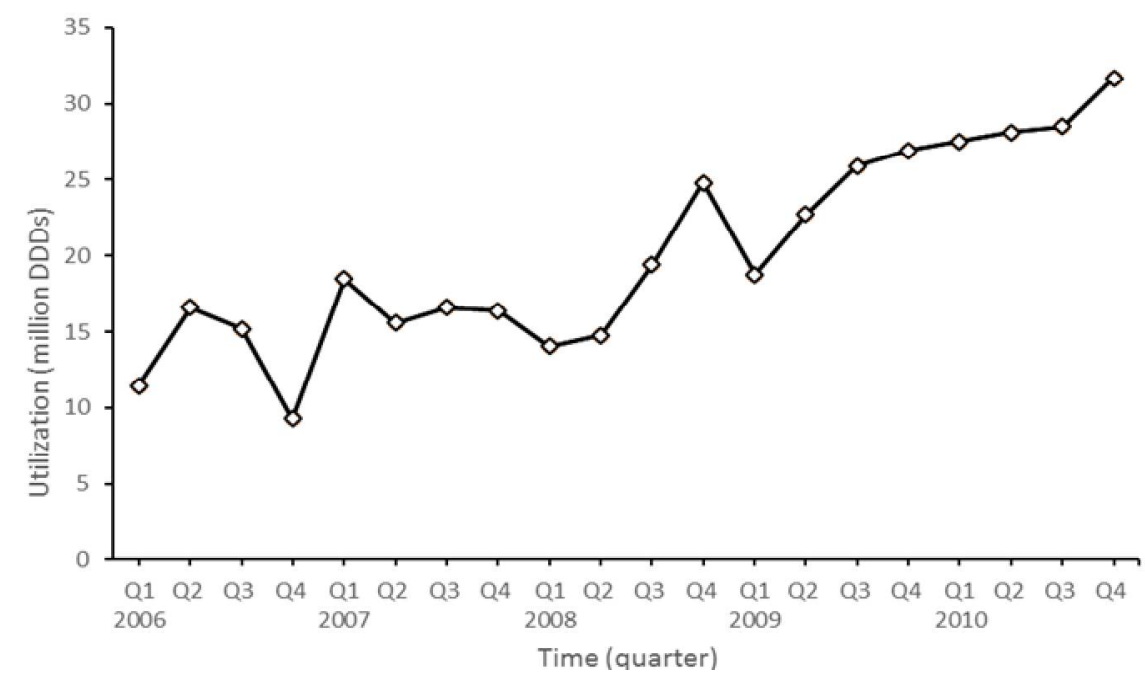

Figure 1: Quarterly medicine utilization trends in NHIF-Sudan, 2006 to 2010 
Table 1: Annual medicine utilization per ATC main group measured in DDD/1000 inhabitants/day, 2006 - 2010

\begin{tabular}{|c|c|c|c|c|c|c|c|}
\hline \multirow[t]{2}{*}{ S/no. } & \multirow[t]{2}{*}{ ATC/main group } & \multicolumn{6}{|c|}{ Utilization in DDD/1000 inhabitants/day } \\
\hline & & 2006 & 2007 & 2008 & 2009 & 2010 & ${ }^{\star 2} 2006-2010$ \\
\hline 1 & Blood and blood-forming organs & 1.15 & 33.11 & 29.68 & 39.84 & 49.20 & 153.01 \\
\hline 2 & Cardiovascular system & 33.15 & 20.00 & 20.16 & 17.67 & 23.20 & 114.18 \\
\hline 3 & General anti-infectives for systemic use & 14.53 & 15.89 & 14.9 & 14.3 & 18.70 & 78.34 \\
\hline 4 & Alimentary tract and metabolism & 15.34 & 12.38 & 9.33 & 10.53 & 12.70 & 60.27 \\
\hline 5 & Musculo-skeletal system & 0.06 & 8.57 & 7.14 & 7.47 & 9.10 & 32.32 \\
\hline 6 & Sensory organs & 16.44 & 4.03 & 1.71 & 3.82 & 4.57 & 30.56 \\
\hline 7 & Respiratory system & 3.87 & 4.73 & 4.36 & 4.58 & 5.40 & 22.93 \\
\hline 8 & Anti-parasitic products, insecticides and repellents & 7.46 & 5.07 & 3.16 & 2.95 & 3.34 & 21.97 \\
\hline 9 & Nervous system & 2.73 & 3.41 & 2.34 & 3.04 & 3.60 & 15.13 \\
\hline 10 & Genito-urinary system and sex hormones & 1.75 & 2.24 & 2.8 & 2.27 & 2.46 & 11.51 \\
\hline 11 & $\begin{array}{l}\text { Systemic hormonal preparations, excluding sex } \\
\text { hormones }\end{array}$ & 1.02 & 2.19 & 1.99 & 1.71 & 1.92 & 8.83 \\
\hline 12 & Dermatologicals & 0.01 & 0.07 & 0.07 & 0.04 & 0.05 & 0.23 \\
\hline 13 & Antineoplastic and immuno-modulating agents & 0.00 & 0.06 & 0.05 & 0.05 & 0.03 & 0.19 \\
\hline Total & & 97.51 & 111.73 & 97.68 & 108.25 & 134.00 & 549.48 \\
\hline
\end{tabular}

Table 2: Contribution of ATC main groups to total medicine utilization in NHIF-Sudan, 2006-2010

\begin{tabular}{|c|c|c|c|c|c|c|c|c|}
\hline & & 2006 & & 2010 & & $2006-2010$ & & \\
\hline $\mathrm{S} / \mathrm{n}$ & ATC/main group & $\begin{array}{l}\text { Utilization in } \\
\text { DDD/1000 } \\
\text { Inhabitants/day }\end{array}$ & $\begin{array}{l}\% \text { of } \\
\text { total }\end{array}$ & $\begin{array}{l}\text { Utilization in } \\
\text { DDD/1000 } \\
\text { Inhabitants/day }\end{array}$ & $\begin{array}{l}\% \text { of } \\
\text { total }\end{array}$ & $\begin{array}{c}\text { Utilization in } \\
\text { DDD/1000 } \\
\text { Inhabitants/day } \\
\text { change }\end{array}$ & $\begin{array}{c}\% \text { of total } \\
\text { utilization } \\
\text { change }\end{array}$ & $\begin{array}{c}{ }^{*} \text { Average } \\
\text { annual } \\
\text { growth } \\
\text { rate } \\
\end{array}$ \\
\hline 1 & $\begin{array}{l}\text { Alimentary tract and } \\
\text { metabolism }\end{array}$ & 15.34 & 15.73 & 12.69 & 9.45 & -2.65 & -7.20 & -4.63 \\
\hline 2 & $\begin{array}{l}\text { Blood and blood-forming } \\
\text { organs }\end{array}$ & 1.15 & 1.18 & 49.22 & 36.65 & 48.1 & 130.66 & 155.78 \\
\hline 3 & Cardiovascular system & 33.15 & 34.00 & 23.2 & 17.27 & -9.95 & -27.05 & -8.54 \\
\hline 4 & Dermatologicals & 0.01 & 0.01 & 0.05 & 0.04 & 0.04 & 0.11 & 49.53 \\
\hline 5 & $\begin{array}{l}\text { Genito-urinary system and sex } \\
\text { hormones }\end{array}$ & 1.75 & 1.79 & 2.46 & 1.83 & 0.71 & 1.93 & 8.89 \\
\hline 6 & $\begin{array}{l}\text { Systemic hormonal } \\
\text { preparations, excluding sex } \\
\text { hormones }\end{array}$ & 1.02 & 1.05 & 1.92 & 1.43 & 0.90 & 2.45 & 17.13 \\
\hline 7 & $\begin{array}{l}\text { General anti-infectives for } \\
\text { systemic use }\end{array}$ & 14.53 & 14.90 & 18.73 & 13.95 & 4.2 & 11.42 & 6.55 \\
\hline 8 & $\begin{array}{l}\text { Antineoplastic and immuno- } \\
\text { modulating agents }\end{array}$ & 0.00 & 0.00 & 0.03 & 0.02 & 0.03 & 0.08 & 0.00 \\
\hline 9 & Musculo-skeletal system & 0.06 & 0.06 & 9.1 & 6.78 & 9.04 & 24.57 & 250.93 \\
\hline 10 & Nervous system & 2.73 & 2.80 & 3.6 & 2.68 & 0.87 & 2.36 & 7.16 \\
\hline 11 & $\begin{array}{l}\text { Anti-parasitic products, } \\
\text { insecticides and repellents }\end{array}$ & 7.46 & 7.65 & 3.34 & 2.49 & -4.12 & -11.20 & -18.2 \\
\hline $\begin{array}{l}12 \\
13\end{array}$ & $\begin{array}{l}\text { Respiratory system } \\
\text { Sensory organs }\end{array}$ & $\begin{array}{c}3.87 \\
16.44\end{array}$ & $\begin{array}{c}3.97 \\
16.86\end{array}$ & $\begin{array}{l}5.40 \\
4.57\end{array}$ & $\begin{array}{l}4.02 \\
3.40\end{array}$ & $\begin{array}{r}1.53 \\
-11.9\end{array}$ & $\begin{array}{c}4.16 \\
-32.26\end{array}$ & $\begin{array}{l}-27.37 \\
0.63\end{array}$ \\
\hline Tot & & 97.51 & 100.00 & 134.3 & 100.00 & 36.80 & 100.00 & 8.33 \\
\hline
\end{tabular}

${ }^{*}$ The percentage may not equal 100 due to rounding

\section{(a) Medicine utilization for second-level therapeutic subgroup}

Medicine utilization analysis was further broken down by second-level ATC classification. The second level of the ATC classification groups medicines of different pharmacological classes that have the same main therapeutic use. Table 3 displays the top 12 therapeutic classes based on their level of utilization (DDD/1000/inhabitant /day). They represent approximately $19.05 \%$ of the total number of therapeutic classes (at the second level), and accounted for $84.02 \%$ of total medicine utilization for the period from 2006 to 2010. The medicine classes that accounted for the highest utilization were antianaemic preparations (32.59 \%), followed by antibacterials for systemic use with $16.81 \%$ and beta blocking agents (7.30 \%). 
Table 3: Medicine utilization of top 12 second-level therapeutic subgroups, based on retail prices (RP) SDG, 2006-2010

\begin{tabular}{|c|c|c|c|c|c|c|c|c|}
\hline S/no. & $\begin{array}{l}\text { ATC } \\
\text { code }\end{array}$ & Description & 2006 & 2007 & 2008 & 2009 & 2010 & *2006-2010 \\
\hline 1 & B03 & Antianaemic preparations & 0.01 & 32.72 & 29.39 & 39.41 & 48.92 & 150.45 \\
\hline 2 & $\mathrm{~J} 01$ & Antibacterials for systemic use & 14.52 & 15.73 & 14.71 & 14.12 & 18.54 & 77.61 \\
\hline 3 & $\mathrm{C} 07$ & Beta blocking agents & 7.67 & 6.89 & 6.9 & 6.05 & 6.23 & 33.73 \\
\hline 4 & M01 & $\begin{array}{l}\text { Anti-inflammatory and Antirheumatic } \\
\text { products }\end{array}$ & 0.06 & 8.19 & 6.65 & 7.11 & 8.68 & 30.69 \\
\hline 5 & S01 & Ophthalmologicals & 16.44 & 4.03 & 1.71 & 3.82 & 4.57 & 30.56 \\
\hline 6 & $\mathrm{C} 09$ & $\begin{array}{l}\text { Agents acting on the renin-angiotensin } \\
\text { system }\end{array}$ & 16.05 & 3.65 & 3.17 & 3.53 & 4.17 & 30.56 \\
\hline 7 & $\mathrm{C} 08$ & Calcium channel blockers & 1.63 & 5.54 & 5.99 & 4.56 & 8.31 & 26.04 \\
\hline 8 & A02 & Drugs for acid-related disorders & 7.71 & 3.37 & 2.77 & 3.06 & 3.66 & 20.57 \\
\hline 9 & A10 & Drugs used in diabetes & 6.66 & 3.51 & 2.89 & 3.37 & 4.07 & 20.49 \\
\hline 10 & P01 & Antiprotozoals & 7.1 & 4.8 & 2.72 & 2.56 & 3.03 & 20.22 \\
\hline 11 & R06 & Antihistamines for systemic use & 1.08 & 2.41 & 2.39 & 2.46 & 2.66 & 11.01 \\
\hline 12 & A03 & $\begin{array}{l}\text { Drugs for functional gastrointestinal } \\
\text { disorders }\end{array}$ & 0.69 & 2.53 & 1.74 & 2.27 & 2.55 & 9.79 \\
\hline Total & & & 79.62 & 93.37 & 81.03 & 92.32 & 115.39 & 461.7 \\
\hline
\end{tabular}

${ }^{*}$ Medicines sorted in descending order, 2006-2010

Table 4 shows the expenditure change between 2006 and 2010 and the percentage contribution of second-level therapeutic subgroups to total medicine utilization.

The top 12 therapeutic classes experienced an average annual utilization growth rate of $37.68 \%$ between 2006 and 2010. Among the top three ATC level 2 therapeutic classes analysed were: antianaemic preparations made the largest contribution to utilization growth (132.94\%), followed by anti-inflammatory and antirheumatic products $(23.43 \%)$, followed by calcium channel blockers (18.16\%).In terms of average annual utilization growth rates, antianaemic had the highest average $(736.32 \%)$ and antiprotozoals the lowest (-19.17\%) (Table 4).

\section{(b) Utilization analysis for the top leading therapeutic groups ATC second-level}

This section provides further analysis for the top three ATC level-2 therapeutic classes, which make a substantial contribution to total medicine utilization, as, identified in previous analysis. For each class the top three medicines by fifth level were determined.

\section{(i) Antianaemic preparations}

Medicine utilization in this therapeutic class had a relatively high percentage share of total utilization for 2006 to 2010 (32.59

$\%)$. Utilization in this therapeutic class increased from 0.01DID in 2006 to 48.92 DID in 2010, with an annual average growth rate of $736.32 \%$ (Figure 2).

The top three medicine products within this therapeutic class were folic acid $5 \mathrm{mg}$ tab (127.06 DID), ferrous sulphate + folic acid $150+0.5 \mathrm{mg}$ cap (17.45 DID) and ferrous sulphate $200 \mathrm{mg}$ tab (2.80 DID) (Table 5).

\section{(ii) Anti-inflammatory and antirheumatic products}

Medicine utilization in this therapeutic class had a relatively high percentage share (in 2006 to $2010,16.81 \%$ of total utilization). Utilization in this therapeutic class increased from 0.06 DID in 2006 to 8.68 DID in 2010, with an annual average growth rate of $246.81 \%$ (Figure 3 ).

The top three medicine products within this therapeutic class were mefenamic acid $500 \mathrm{mg}$ tab (11.10 DID), diclofenac Sodium $100 \mathrm{mg}$ tab (8.72 DID) and diclofenac sodium 25mg tab (3.55 DID) (Table 6).

\section{(iii) Calcium channel blockers}

Medicine utilization in this therapeutic class had 
Table 4: Percentage contribution of top 12 second-level therapeutic subgroups to total medicine utilization in NHIF-Sudan, 2006-2010

\begin{tabular}{|c|c|c|c|c|c|c|c|c|c|}
\hline & \multirow[b]{2}{*}{$\begin{array}{l}\text { ATC second } \\
\text { level }\end{array}$} & \multirow[b]{2}{*}{ Description } & \multicolumn{2}{|l|}{2006} & \multicolumn{2}{|l|}{2010} & \multicolumn{2}{|c|}{$2006-2010$} & \multirow[b]{2}{*}{$\begin{array}{c}\text { Average } \\
\text { annual } \\
\text { growth rate } \\
(\%)\end{array}$} \\
\hline & & & $\begin{array}{l}\text { Utilization in DDD/1000 } \\
\text { Inhabitants/day }\end{array}$ & $\begin{array}{l}\% \text { of } \\
\text { total }\end{array}$ & $\begin{array}{c}\text { Utilization in } \\
\text { DDD/1000 } \\
\text { Inhabitants/day }\end{array}$ & $\begin{array}{l}\% \text { of } \\
\text { total }\end{array}$ & $\begin{array}{l}\text { Utilization } \\
\text { change }\end{array}$ & $\begin{array}{c}\text { Total } \\
\text { utilization } \\
\text { change }(\%)\end{array}$ & \\
\hline 1 & B03 & Antianaemic preparations & 0.01 & 0.01 & 48.92 & 36.43 & 48.91 & 132.94 & 736.32 \\
\hline 2 & $\mathrm{~J} 01$ & Antibacterials for systemic use & 14.52 & 14.89 & 18.54 & 13.80 & 4.02 & 10.93 & 6.3 \\
\hline 3 & M01 & $\begin{array}{l}\text { Anti-inflammatory and } \\
\text { antirheumatic products }\end{array}$ & 0.06 & 0.06 & 8.68 & 6.46 & 8.62 & 23.43 & 246.81 \\
\hline 4 & $\mathrm{C08}$ & Calcium channel blockers & 1.63 & 1.67 & 8.31 & 6.19 & 6.68 & 18.16 & 50.26 \\
\hline 5 & $\mathrm{C} 07$ & Beta blocking agents & 7.67 & 7.87 & 6.23 & 4.64 & -1.44 & -3.91 & -5.07 \\
\hline 6 & S01 & Ophthalmologicals & 16.44 & 16.86 & 4.57 & 3.40 & -11.87 & -32.26 & -27.39 \\
\hline 7 & C09 & $\begin{array}{l}\text { Agents acting on the renin- } \\
\text { angiotensin system }\end{array}$ & 16.05 & 16.46 & 4.17 & 3.10 & -11.88 & -32.29 & -28.61 \\
\hline 8 & A10 & Drugs used in diabetes & 6.66 & 6.83 & 4.07 & 3.03 & -2.59 & -7.04 & -11.58 \\
\hline 9 & $\mathrm{~A} 02$ & Drugs for acid-related disorders & 7.71 & 7.91 & 3.66 & 2.73 & -4.05 & -11.01 & -16.99 \\
\hline 10 & P01 & Antiprotozoals & 7.10 & 7.28 & 3.03 & 2.26 & -4.07 & -11.06 & -19.17 \\
\hline 11 & R06 & Antihistamines for systemic use & 1.08 & 1.11 & 2.66 & 1.98 & 1.58 & 4.29 & 25.28 \\
\hline 12 & $\mathrm{~A} 03$ & $\begin{array}{l}\text { Drugs for functional } \\
\text { gastrointestinal disorders }\end{array}$ & 0.69 & 0.71 & 2.55 & 1.90 & 1.86 & 5.06 & 38.65 \\
\hline $\begin{array}{l}\text { Subtotal- } \\
\text { utilization }\end{array}$ & $\begin{array}{c}2 \text { level top } \\
12 \\
\end{array}$ & & 79.62 & 81.65 & 115.39 & 85.92 & 35.77 & 97.23 & 9.72 \\
\hline $\begin{array}{c}\text { Total } \\
\text { medicines } \\
\text { utilization }\end{array}$ & & & 97.51 & 100 & 134.30 & 100 & 36.79 & 100.00 & 8.33 \\
\hline
\end{tabular}

${ }^{*}$ Percentage may not equal 100 due to rounding up 


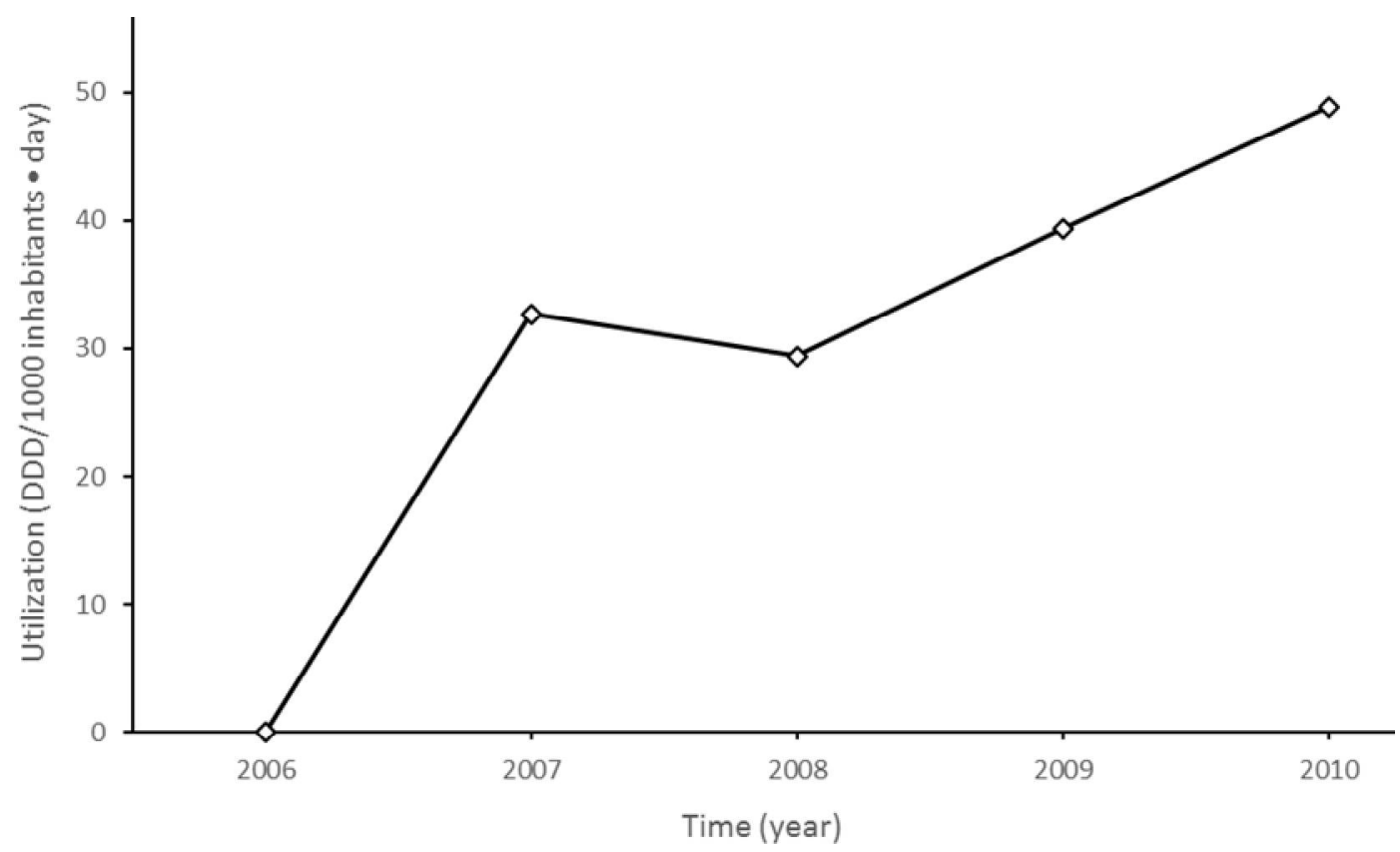

Figure 2: Antianaemic preparations: utilization trend in NHIF-Sudan, 2006 to 2010

Table 5: Antianaemic preparations utilization in NHIF-Sudan based on retail prices (RP) SDG, 20062010

\begin{tabular}{|c|c|c|c|c|c|c|c|}
\hline \multirow[b]{2}{*}{$\mathbf{S} / \mathbf{N}$} & \multirow[b]{2}{*}{ Drug name (generic) } & \multicolumn{6}{|c|}{ Utilization in DDD/1000 inhabitants/day } \\
\hline & & 2006 & 2007 & 2008 & 2009 & 2010 & $\begin{array}{l}* 2006- \\
2010\end{array}$ \\
\hline \multirow[t]{2}{*}{1} & Folic acid $5 \mathrm{mg}$ tab & & 27.2 & 23.2 & 34.6 & 41.8 & 127.0 \\
\hline & & 0.00 & 9 & 3 & 9 & 5 & 6 \\
\hline 2 & $\begin{array}{l}\text { Ferrous sulphate + Folic acid } 150+0.5 \mathrm{mg} \\
\text { cap }\end{array}$ & 0.00 & 3.68 & 4.65 & 4.18 & 4.94 & 17.45 \\
\hline \multirow{2}{*}{$\begin{array}{l}3 \\
4\end{array}$} & Ferrous sulphate $200 \mathrm{mg}$ tab & 0.00 & 1.00 & 0.82 & 0.23 & 0.74 & 2.80 \\
\hline & $\begin{array}{l}\text { Vitamin B12 (cyanocobalamine) 1mg/ml } \\
\text { (Inj) }\end{array}$ & 0.00 & 0.52 & 0.34 & 0.00 & 0.91 & 1.78 \\
\hline 5 & Ferrous sulphate 50 mg syrup & 0.00 & 0.15 & 0.19 & 0.13 & 0.19 & 0.66 \\
\hline 6 & Vitamin B12 (cyanocobalamine) tab & 0.00 & 0.06 & 0.09 & 0.15 & 0.12 & 0.41 \\
\hline 7 & Erythropoitin inj 4000I.U (Inj) & 0.01 & 0.02 & 0.07 & 0.00 & 0.16 & 0.25 \\
\hline \multirow[t]{2}{*}{8} & Iron dextran $50 \mathrm{mg} / \mathrm{ml}$ (Inj) & 0.00 & 0.00 & 0.01 & 0.03 & 0.01 & 0.04 \\
\hline & & & 32.7 & 29.4 & 39.4 & 48.9 & 150.4 \\
\hline Total & & 0.01 & 2 & 0 & 1 & 2 & 5 \\
\hline
\end{tabular}

${ }^{*}$ Medicines sorted in descending order, 2006-2010 


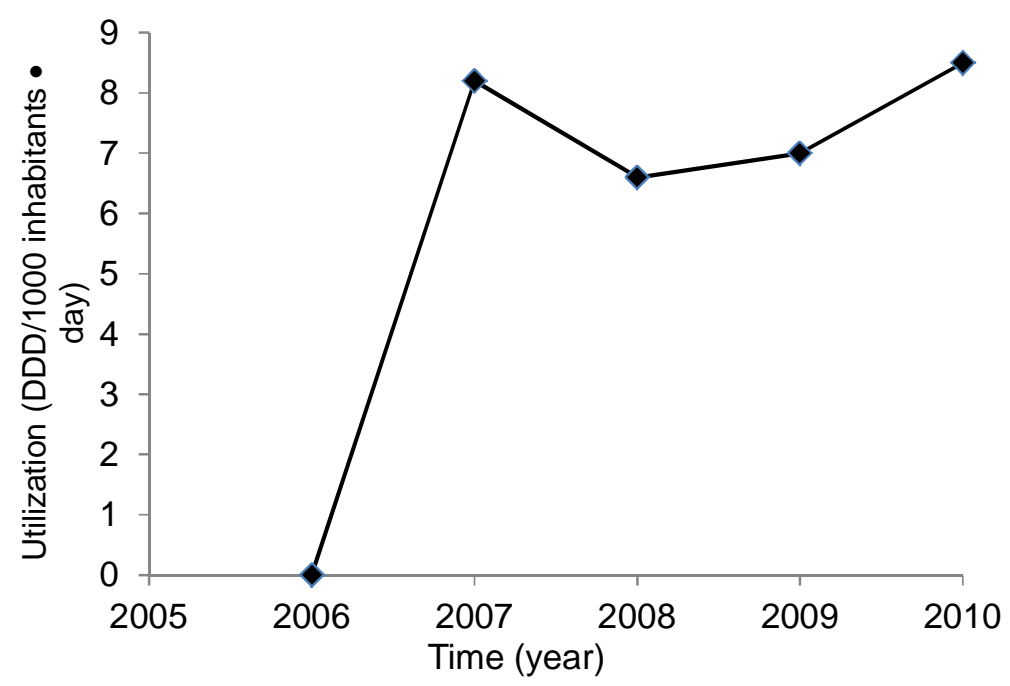

Figure 3: Anti-inflammatory and antirheumatic products trend in NHIF-Sudan, 2006 to 2010

Table 6: Medicine utilization of anti-inflammatory and antirheumatic products based on retail prices (RP) SDG, 2006-2010

\begin{tabular}{llcccccc}
\hline & & \multicolumn{5}{c}{ Utilization in DDD/1000 } & inhabitants/day \\
\cline { 3 - 7 } & Medicinename (generic) & $\mathbf{2 0 0 6}$ & $\mathbf{2 0 0 7}$ & $\mathbf{2 0 0 8}$ & $\mathbf{2 0 0 9}$ & $\mathbf{2 0 1 0}$ & ${ }^{\star} \mathbf{2 0 0 6 - 2 0 1 0}$ \\
\hline 1 & Mefenamic acid 500mg tab & 0.01 & 2.43 & 2.38 & 1.72 & 2.17 & 11.10 \\
2 & Diclofenac Sodium 100mg tab & 0.01 & 1.06 & 0.71 & 0.86 & 0.92 & 8.72 \\
3 & Diclofenac Sodium 25mg tab & 0.03 & 0.09 & 0.09 & 0.25 & 0.35 & 3.55 \\
4 & Mefenamic acid 250mg tab & 0.01 & 0.42 & 0.14 & 0.31 & 0.39 & 2.93 \\
5 & Diclofenac Sodium 75 mg (Inj) & 0.00 & 0.18 & 0.13 & 0.13 & 0.15 & 1.28 \\
6 & Ibuprofen 400mg tab & 0.00 & 0.37 & 0.08 & 0.25 & 0.35 & 1.05 \\
7 & Diclofenac Sodium 50mg tab & 0.00 & 0.03 & 0.02 & 0.03 & 0.03 & 0.81 \\
8 & Ibuprofen 200mg tab & 0.00 & 0.00 & 0.01 & 0.00 & 0.01 & 0.59 \\
9 & Indomethazine 25mg cap & 0.00 & 0.17 & 0.12 & 0.12 & 0.12 & 0.53 \\
10 & Ibuprofen 600 mg tab & 0.00 & 0.70 & 0.59 & 0.68 & 0.96 & 0.12 \\
11 & Indomethacine 100 mg susp & 0.00 & 2.73 & 2.38 & 2.76 & 3.22 & 0.02 \\
12 & Mefenamic acid 50mg/5ml susp & 0.00 & 0.00 & 0.00 & 0.00 & 0.01 & 0.01 \\
\hline Total & & $\mathbf{0 . 0 6}$ & $\mathbf{8 . 1 8}$ & $\mathbf{6 . 6 5}$ & $\mathbf{7 . 1 2}$ & $\mathbf{8 . 6 8}$ & $\mathbf{3 0 . 7 1}$ \\
\hline
\end{tabular}

${ }^{*}$ Medicines sorted in descending order, 2006-2010

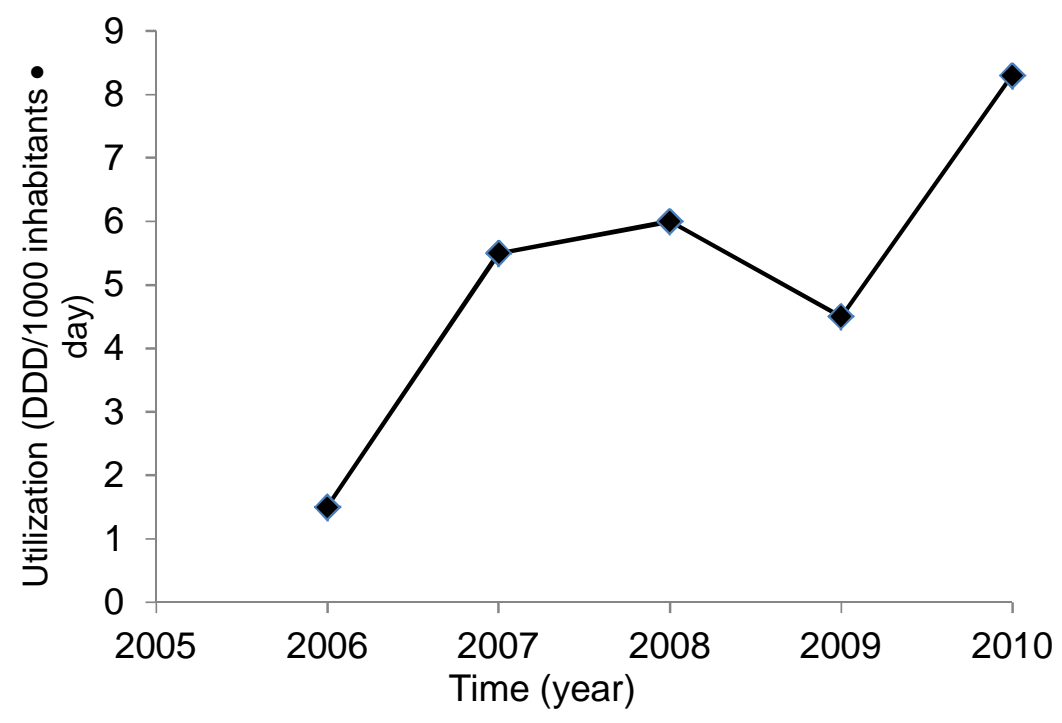

Figure 4: Calcium channel blockers utilization trend in NHIF-Sudan, 2006 to 2010 
Table 7: Calcium channel blockers utilization, based on retail prices (RP) SDG, 2006-2010

S/no. Drug name (generic) Utilization DDD/1000/day

\begin{tabular}{llcccccc}
\cline { 3 - 7 } & & $\mathbf{2 0 0 6}$ & $\mathbf{2 0 0 7}$ & $\mathbf{2 0 0 8}$ & $\mathbf{2 0 0 9}$ & $\mathbf{2 0 1 0}$ & *2006-2010 \\
\hline 1 & Amlodipine 5mg tab & 1.33 & 2.27 & 2.29 & 0.81 & 3.77 & 10.47 \\
2 & Amlodipine 10mg tab & 0.08 & 0.57 & 1.30 & 2.27 & 3.34 & 7.56 \\
3 & Nifedipine 20mg tab & 0.00 & 1.80 & 1.87 & 1.08 & 0.83 & 5.57 \\
4 & Nifedipine 10mg tab & 0.00 & 0.51 & 0.31 & 0.22 & 0.14 & 1.18 \\
5 & Felodipine 5mg tab & 0.22 & 0.36 & 0.20 & 0.17 & 0.22 & 1.16 \\
6 & Diltiazem HCL 60mg tab & 0.01 & 0.03 & 0.02 & 0.02 & 0.01 & 0.09 \\
\hline Total & & $\mathbf{1 . 6 4}$ & $\mathbf{5 . 5 4}$ & $\mathbf{5 . 9 9}$ & $\mathbf{4 . 5 7}$ & $\mathbf{8 . 3 1}$ & $\mathbf{2 6 . 0 3}$ \\
\hline
\end{tabular}

${ }^{*}$ Medicines sorted in descending order, 2006-2010

a relatively high percentage share (for 2006$2010,5.64 \%$ of total utilization). Utilization in this therapeutic class increased from 1.63 DID in 2006 to 8.31 DID in 2010, with an annual average growth rate of $50.26 \%$ (Figure 4 ).

The top three medicine products within this therapeutic class were amlodipine $5 \mathrm{mg}$ tab (10.47 DID), amlodipine $10 \mathrm{mg}$ tab (7.56 DID) and nifedipine $20 \mathrm{mg}$ tab (5.57 DID) (refer to Table 7).

\section{Correlations between medicine utilization and population coverage per quarter 2006-2010}

Pearson correlation analysis indicated that medicine utilization (DDDs) showed a very weak positive correlation with population coverage per quarter $(r=0.161, p<0.001)$.

Correlations between medicine utilization and number of patients per quarter 20062010

Pearson correlation analysis also showed a very weak positive correlation between medicine utilization (DDDs) with the number of patients (beneficiaries) $(r=0.090, p<0.001)$.

\section{Differences in medicine utilization between years}

One-way ANOVA was used to examine the differences between medicine utilization among years. There was a statistically significant difference between groups $\{F(4,5270)=54.412$, $p<0.001\}$. Tukey post-hoc test revealed that the observed significant difference between the mean medicine utilization among the years lies between mean proportional medicine utilization for the years 2006 $(M=7.77, S D=3.17)$ and 2010 $(\mathrm{M}=9.43, \mathrm{SD}=0.03)(p<0.001)$. Other differences were not significant.

\section{Differences in medicine utilization between quarters}

To examine the differences between medicine utilization between quarters, one-way ANOVA was used.There was no statistically significant difference between groups $\{F(3,5271)=1.297$, $p=0.273\}$.

Differences in medicine utilization between ATC main groups in different quarters

Factorial design two-way ANOVA was used to compare differences in mean medicine utilization between ATC main groups for different quarters. There was no significant differences between the ATC main groups in different quarters $\{F(36$, 5275) $=0.183, p=1.000\}$.

\section{Differences in medicine utilization between $A B C$ classes in different quarters 2006-2010}

There was no significant differences showed by factorial design two-way ANOVA between the ATC main groups in different quarters $(F(6$, $5275)=1.786, p=0.098$ ).

\section{Differences in medicine utilization between VEN groups in different quarters 2006-2010}

Factorial design two-way ANOVA showed no significant differences between the ATC main groups indifferent quarters $\{F(6,5275)=0.116$, $p=0.995\}$.

\section{DISCUSSION}

This study presented comprehensive descriptive and comparative medicine utilization data for a national health insurance scheme in Sudan between 2006 and 2010 using a standard recommended method (DDD methodology) which is a powerful tool for medicine utilization research. The results are useful for policy- 
makers in the planning and assessment of medicine policies to achieve health goals.

The findings indicate a significant growth in medicine utilization in terms of DDDs, reflecting both demand and supply sides. This growth is most likely due to increases in medicine prices, high utilization rate by patients or expansion of insurance coverage. Since 2007, the NHIF formulary has been expanded to include generic and innovative medicines. Spending was driven by higher utilization of existing and newer medicines [8].Statistically, the trend of medicine utilization with regard to ATC classes, ABC classes and VEN show that the classes were no statistically significant differences across quarters. The findings indicate statistically significant difference in medicine utilization across years. The difference was however, statistically significant lower in 2006 and 2010.

There were no significant differences in medicine utilization between years in general; medicine utilization was largely the same between the years because of the formulary list change in 2007 and the intensive regulations enacted during this period [9]. A positive medicines list was available in NHIF-Sudan since 1996. Interbrand competition was introduced in NHIF-Sudan since 2002. This has successfully decreased the medicines prices to about two-fifth of the tender fund if the bases was the wholesale prices. In addition, it was found that medicine utilization has a significantly stronger correlation with number of population covered per year quarter than the number of patient visits per year quarter. This indicates that the high utilization rate depends on insurance coverage rather than frequency of patient visits.

High medicine utilization was explained by four therapeutic classes, i.e., blood and blood-forming organs (B), the cardiovascular system (C), general anti-infectives for systemic use $(\mathrm{J})$, and the alimentary tract and metabolism (A); all were responsible for most of the medicine utilization. Two of them ( $B$ and $\mathrm{J}$ ) in addition to the musculoskeletal system (M) and respiratory system (R) were responsible for most of the contribution to total increases in medicine utilization between 2006 and 2010. The other two classes (C) and (A) showed a significant fall between 2006 and 2010. Musculo-skeletal system (M) showed significant increase in the same period and is one of the classes that made a high contribution to total utilization increase and was the fastest growing medicine class (an average of 250.93 per cent per year). The fastest declining medicine class, in terms of medicine utilization, was systemic hormonal preparations, excluding sex hormones $(\mathrm{H})$ (decreased by an average of $8.57 \%$ per year); probably in response to new restrictions imposed on the NHIF formulary in 2007 [8].

In the second-level therapeutic subgroup, the classes that accounted for the highest utilization were antiemetic preparations (B03), followed by antibacterials for systemic use (J01) and beta blocking agents (C07). Only one of them (B03) in addition to anti-inflammatory and antirheumatic products (M01) and calcium channel blockers (C08) was responsible for the largest contribution to total utilization increases between 2006 and 2010. The fastest growing drug class was anti-anaemic preparations and the fastest declining drug class, in terms of drug expenditure, was antiprotozoals. When analysing the correlation between these results and the morbidity and mortality trends in Sudan we found that the same pattern of diseases was reflected, as malnutrition, acute renal failure, malignant neoplasm, heart failure, pneumonia, and septicaemia; all are in the top 10 causes of mortality in hospitals [10].

International comparisons for medicine utilization are difficult, owing to differences in healthcare systems, culture and politics [11].

Most of the developing countries do not publish national statistics on drug use, and pharmaceutical companies maintain information private for trade reasons [12]. A survey used data from five African countries: Gambia, Ghana, Kenya, Nigeria and Uganda confirmed that antibiotics were widely available and inappropriately used in all settings [13]. Unfortunately, individual medications were not measured using same methods that were used in this study [2,3]. Only one study was found in the regions that could be used for comparing antibacterials for systemic use with the current results. Although NHIF-Sudan is not representative of the whole country, it can offer a general perspective as it covers about two-fifth of the Sudanese population; the results of the study show the general medicine trend in the country and could be used for future research.

In Sudan, antianaemic drugs are usually prescribed for pregnant mothers although they are also used to treat anaemia resulting from treatment of cancer. In this study, folic acid tablet was the most common medicine used in this class. Nordic countries had a low utilization rate of antianaemic preparations [14]. In Australia, hydroxocobalamin was the medicine used the most in 2006 and 2007. There was slight increase $(26 \%)$ in anti-anaemic preparation 
utilization in 2006 (7.25 DID) and 2007 (9.18 DID) [15] as compared with the present study (0.01 DID (in 2006) and 32.72 DID (in 2007)); $327100 \%)$. In Malaysia, the anti-anaemic preparation utilization for 2006 (0.18 DID) and 2007 (0.16 DID) was generally lower than the present study. The most commonly prescribed anti-anaemics in Malaysia in 2007 were erythropoietin injections $(0.156$ DDD/1000 population/day) [16] and this was lower than in NHIF-Sudan.

In Tanzania, antibacterial utilization in 2009 (4.99 DID) was lower than in the present study [17]. In another finding, the antibacterial utilization in 2006 and 2007 was almost $50 \%$ less than in the present study [16]. Meanwhile, in Nordic countries, the average DID of utilization of antibacterials for systemic use was higher [14].In Australia, amoxicillin was the most widely used medicine in 2006 and 2007 (16.27 DID and 23.91 DID, respectively). Antibacterial utilization in 2006 and 2007 was higher than the present study, respectively [15].In Malaysia, the most commonly used antibacterials for systemic use was ampicillin whereas amoxicillin was the one most widely used in NHIF-Sudan and Tanzania.

In Australia, atenolol was the most widely used beta blocker in 2006 and 2007, similarly to Malaysia and NHIF-Sudan. However, beta blocker utilization in 2006 and 2007 in Australia (26.58 DID and 26.83 DID), Nordic countries (46.26 DID and 46.30 DID) and Malaysia (25.71DID and 26.26 DID) was much higher than in the present study [14-16].

Previous findings indicated that a small number of items are responsible for largepercentage of the total NHIF budget [18], whereas around onefourth of number of items were responsible for only a small percentage of expenditure from the total budget [18].From the international comparison, it can be concluded that NHIFSudan had higher utilization rate of antianaemic preparations compared with Malaysia, Australia and Nordic countries. Furthermore, NHIF-Sudan had a high utilization rate of antibacterials for systemic use compared with Malaysia and a low rate when compared to Australia and Nordic countries. Although there is high utilization rate of antibacterials for systemic use in developed countries, this can be explained by the large number of chemical entries in these countries compared to the developing countries where the utilization is restricted to narrow spectrum level of treatment [19]. In contrast, the present study also found more use of second and third line antibacterial treatment as compared to developed countries where they are more towards first-line of treatment. On the other hand, NHIF-Sudan had a lower utilization rate of beta blocking agents compared with Australia, Nordic countries and Malaysia.

\section{Limitations of the study}

There is a dearth of similar studies in the developing and low-and middle-income countries. Thus, it was difficult to compare these present study findings with others. In addition, the medicine use data for individual patient (prescriptions) was not available in NHIF databases. Only aggregate medicine data (spending, quantities and prices) was obtained from NHIF-Sudan headquarters. Therefore, medicine use variation by demographic characteristics (age-sex) was not captured in this study. A last point that is worth mentioning is the trend of drug utilization studied that was for the years 2006 - 2010. The findings are still important and useful. The list of medicines is still been used till today. No much changes in the policy and guidelines. These findings could be used for further improvement in practice and research.

\section{CONCLUSION}

The findings of this study show that the total medicine utilization between 2006 and 2010 significantly increased. The medicine classes that accounted for the highest utilization were medicines related to blood and blood-forming organs, followed by those related to the cardiovascular system, the general anti-infectives for systemic use and the alimentary tract and metabolism. This study provides evidence that all these quantification methods are very useful tools that pharmacy organizations can and should use to assess trends in drug consumption, manage the medicines list, identify medicine use problems and perform comparisons.

\section{DECLARATIONS}

\section{Acknowledgement}

The authors would like to acknowledge that this paper is taken from a part of a PhD thesis conducted at the School of Pharmaceutical Sciences, Universiti Sains Malaysia, Penang, Malaysia, and would also like to thank NHIF Sudan for providing access to the data used in this study. 


\section{Conflict of Interest}

No conflict of interest associated with this work.

\section{Contribution of Authors}

The authors declare that this work was done by the authors named in this article and all liabilities pertaining to claims relating to the content of this article will be borne by them.

\section{Open Access}

This is an Open Access article that uses a funding model which does not charge readers or their institutions for access and distributed under the terms of the Creative Commons Attribution License (http://creativecommons.org/licenses/by/ 4.0) and the Budapest Open Access Initiative (http://www.budapestopenaccessinitiative.org/rea d), which permit unrestricted use, distribution, and reproduction in any medium, provided the original work is properly credited.

\section{REFERENCES}

1. WHO. Country Pharmaceutical Situations: Fact Book on WHO Level I Indicators 2007. (WHO/EMP/MPC/2010.1) World Health Organisation, Geneva, 2010.

2. WHO. Introduction to Drug Utilization Research. WHO International Working Group for Drug Statistics Methodology, WHO Collaborating Center for Drug Statistics Methodology, WHO Collaborating Center for Drug Utilization Research Clinical Pharmacological Services. 2003 [cited 2015 Sept 28]. Available from: http://books.google.com.my/books?id=8ivwtgAACAAJ.

3. WHO. Guidelines for ATC Classification and DDD Assignment. Collaborating Centre for Drug Statistics Methodology. Oslo, 2008.

4. WHO. Studies in Medicine Utilization: Methods and Application. Copenhagen, European Regional Publication Series No. 8, 1979

5. Hutchinson JM, Patrick DM, Marra F, Ng H, Bowie WR, Heule L, Muscat M, Monnet DL. Measurement of antibiotic consumption: A practical guide to the use of the Anatomical Therapeutic Chemical Classification and Defined Daily Dose system methodology in Canada. Can J Infect Dis 2004; 15: 29-35.

6. Polk RE, Fox C, Mahoney A, Letcavage J, MacDougall C. Measurement of adult antibacterial drug use in 130 US hospitals: comparison of defined daily dose and days of therapy. Clin Infect Dis 2007; 44: 664-670.

7. WHO. Everybody Business: Strengthening Health Systems to Improve Health Outcomes: WHO's Framework for Action. Geneva: WHO Library Cataloguing-in-Publication Data, 2007 [cited 2016 March 18]. Available from: http://www.who.int/health systems/strategy/everybodys_business.pdf.
8. NHIF. Pharmaceutical Services Report 2008. Khartoum: Directorate of Pharmaceutical Services, National Health Insurance Fund Sudan, 2009.

9. NHIF. Pharmaceutical Services Report 2010. Khartoum: Directorate of Pharmaceutical Services, National Health Insurance Fund Sudan, 2011.

10. Federal Ministry of Health. Annual Health Statistical Report 2010. Khartoum: National Health Information Centre, Federal Ministry of Health, Sudan, 2011.

11. Australian Institute of Health and Welfare. Statistics on Drug Use in Australia 2004. Canberra: AlHW Australian Institute of Health and Welfare, 2005. [cited 2016 April 10]. Available from: http://www.aihw.gov.au/publicationdetail/?id=6442467739.

12. Istúriz RE, Claude C. Antibiotic use in developing countries. Infect Contr Hosp Epidemiol 2000; 21: 394397.

13. Vialle-Valentin CE, LeCates RF, Zhang F, Desta $A T$, Ross-Degnan D. Predictors of antibiotic use in African communities: evidence from medicines household surveys in five countries. Trop Med Int Health 2012;17(2):211-22. doi: 10.1111/j.1365-3156.2011. 02895.x. Epub 2011 Oct 17.

14. Nordic Medico Statistical Committee. Medicines Consumption in the Nordic Countries 2004-2008. Copenhagen, 2009. [cited 2016 May 10]. Available from: http://nomesco-eng.nom-

nos.dk/filer/publikationer/Medicines\%20consumption\%2 02004-2008\%20web.pdf.

15. Commonwealth of Australia. Australian Statistics on Medicines 2007. Australian Government Department of Health and Ageing, 2009. [cited 2016 May 10]. Available from: http://www.health.gov.au/internet/main/publishing. nsf/Content/A2372BD7A7D36B4BCA2576020014E6C8/ \$File/ASM\%202007\%20-\%20Introduction.pdf.

16. Ministry of Health Malaysia. Malaysian Statistics on Medicine 2007. Kuala Lumpur: Pharmaceutical Services Division \& Clinical Research Centre. The National Medicines Use Survey. Clinical Research Centre, Ministry of Health Malaysia, 2010. [cited 2016 June 10]. Available from: http://www.cornerstone-msc.net/pharm acy_portal/index.cfm?\&menuid=142\&parentid=163.

17. Van Den BJ, Semvua HH, Boeree MJ, Aarnoutse RE, Kibiki GS. Assessment of antibacterial sale in northern Tanzania by using the Anatomic Therapeutic Chemical classification and Defined Daily Dose methodology. Tanz J Health Res 2010;12: 208-212.

18. Mousnad MA, Mohamed Izham MI, Palaian S, Shafie AA. Medicine expenditures in Sudan national health insurance fund: $A n$ ABC-VEN analysis of 5 year medicine consumption. JPHS 2016; 7: 165-171. DOI: 10.1111/jphs. 12136.

19. Ferech M, Coenen S, Malhotra-Kumar S, Dvorakova K, Hendrickx E, Suetens C, Goossens H. European Surveillance of Antimicrobial Consumption (ESAC): outpatient antibiotic use in Europe. J Antimicrob Chemother 2006; 58: 401-407. 
Mousnad et al

Trop J Pharm Res, July 2017; 16(7): 215 